\title{
MANY FACES OF DÉJÀ VU: A NARRATIVE REVIEW
}

\author{
Marija Bošnjak Pašić1, Emina Horvat Velić ${ }^{2}$ Luka Fotak ${ }^{3}$, Hanna Pašićc ${ }^{4}$ Azijada Srkalović Imširagić ${ }^{5}$, \\ Davorka Milat ${ }^{6}$, Helena Šarac ${ }^{7}$, Sarah Bjedov $^{8} \&$ Željka Petelin Gadže ${ }^{9}$ \\ ${ }^{1}$ University Hospital Centre Zagreb, Department of Neurology and School of Medicine, Josip Juraj Strossmayer \\ University of Osijek, Referral Centre of the Ministry of Health of the Republic of Croatia for Demyelinating \\ Diseases of the Central Nervous System, Croatia \\ ${ }^{2}$ University of Zagreb, Faculty of Science, Department of Biology, Zagreb, Croatia (student) \\ ${ }^{3}$ General Hospital Varaždin, Varaždin, Croatia \\ ${ }^{4}$ University of Zagreb, School of Medicine (Medical Studies in English), Zagreb, Croatia (student) \\ ${ }^{5}$ Neuropsychiatric Hospital "Dr Ivan Barbot", Popovača, Croatia \\ "Sveti Rok" Polyclinic, Zagreb, Croatia \\ ${ }^{7}$ University Hospital Centre Zagreb, Department of Neurology, Croatian Institute for Brain Research, \\ School of Medicine, University of Zagreb, Zagreb, Croatia \\ ${ }^{8}$ University Hospital Centre Zagreb, Department of Psychological Medicine, Zagreb, Croatia \\ ${ }^{9}$ University Hospital Centre Zagreb, Department of Neurology, School of Medicine, University of Zagreb, \\ Referral Centre of the Ministry of Health of the Republic of Croatia for Epilepsy, Zagreb, Croatia
}

received: 27.8.2017;

revised: 5.12.2017;

accepted: 10.1 .2018

\section{SUMMARY}

French expression standing for the phrase "already seen" is a déjà vu. It is thought that as much as 97\% of the population have experienced déjà vu at least once in their lifetime and 67\% experience it regularly. The explanations of this phenomenon in novels and poems include reincarnation, dreams, organic factors, and unconscious memories. In this narrative review connection between déjà vu and various other conditions has been mentioned: false memories, temporal lobe epilepsy and other neurological conditions. In psychiatric patients déjà vu phenomenon is more often seen in patients with anxiety and people with derealisation/ depersonalization. It seems that temporal region is the origin of déjà vu phenomena in both healthy individuals and in individuals with neurological and psychiatric conditions, but the exact mechanism of this phenomenon is however still unknown. More attention should also be given to déjà vu from philosophical and religious perspectives as well. Déjà vu is still an enigma which could only be revealed with multidisciplinary approach through cooperation between neurologists, brain scientists, psychiatrists and experimental psychologists.

Key words: anxiety - déjà vu-derealisation - temporal lobe epilepsy

$* * * * *$

\section{INTRODUCTION}

French expression standing for the phrase "already seen" is a déjà vu and it is often regarded as some sort of precognition. However, déjà vu has been revealed as an anomaly related to the memory, with many similar occurrences: déjà entendu (already heard), déjà éprouvé (already experienced, felt, attempted or tried), déjà fait (already done), déjà pensé (already thought), déjà raconté (already recounted, told), déjà senti (already felt emotionally or smelt), déjà su (already known - intellectually), déjà trouvé (already found, met), déjà vécu (already lived through, fully experienced or recollected in its entirety) and déjà voulu (already desired, already wanted) (see Neppe 2015). On the contrary, jamais vu is a phenomenon that is the complete opposite of déjà vu experience (despite the person knowing already being in almost the same situation, they still do not recognize it as such). Somewhat informally, déjà vu could be divided into two forms. First one occurs in healthy people, and second is linked to various psychiatric and neurological condi- tions, with epilepsy being one of the most serious. Those two forms differ in the frequency and length of the experience, where longer déjà vu suggests illness, whether it being neurological or psychological. There are also definitions that differentiate between déjà vu and déjà vecu experiences, with déjà vu defines "erroneous familiarity", and déjà vecu "erroneous recollection" (Illman et al. 2012). Approximately $97 \%$ of the population have experienced déjà vu at least once while rather high number of study participants, $67 \%$, experience it regularly (O'Connor et al. 2008).

The sensation of déjà vu arises as a conjunction of two streams of cognition: the phenomenological experience of recognizing a current situation and the awareness that this feeling of recognition is inappropriate (O'Connor \& Moulin 2010). Déjà vu experience has also been described in many novels and poems. The explanations, formulated by these authors, include reincarnation, dreams, organic factors, and unconscious memories. For déjà vu experiences that are not connected with neurological conditions, psychiatrists and other brain specialists should be encouraged to 
overstep the limits of psychiatric literature and read prose and poetry as well (Sno et al. 1992).

An interesting question arising is whether déjà vu could be nothing more but a by-product of evolution, a survival mechanism that once served a purpose, and is nowadays simply a vestigial remain, like an appendix, somewhat of a "glitch" in the functioning of our mind. Artificially inducing déjà vu could also be used to explore how our brain works in more detail, similar to how visual illusions uncover the way we process information - sometimes, to understand how something works "normally", we have to study it from the perspective of it being "broken". If we dig deeper into our fascination with the phenomenon of consciousness, we inevitably come to questioning the very nature of our reality, since there is a (relatively; in the order of hundreds of milliseconds) significant gap between something happening, that sensory information travelling to the relevant centres of our brain, and only after that it gets integrated into our conscious perception of ourselves and our surroundings. By definition, we are always "lagging" behind the world around us. What if there is a "leak" of information from our unconsciousness into our consciousness "before" we are supposed to retrieve and process that information, a "miswired" brain, in this case the wires being represented by axons, dendrites and neurotransmitters? Could this "miswiring", déjà vu, be a sign of what is next to come in terms of the evolution of our mind? We have a desire to tinker with things, the insatiable urge to learn how the world around us works, to quantify and categorise, to place everything into brackets - a perfectly orchestrated world behaving according to set, rigid, rules. What if our minds cannot be described as computers, linear machines? If we dig into the realm of quantum physics, things become more "fluid", there are no pre-set outcomes, just probabilities of something happening maybe déjà vu is simply quantum world manifesting itself on a larger scale.

Not limiting ourselves to strictly scientific explanations, our fascination with déjà vu can, and should, also be viewed from a religious or spiritual perspective, in which the experience is often attributed to people "connected" to divine entities, ranging from prophets, people who have been reincarnated or even deities themselves. When it comes to pathologies of the brain that alter the persons reality, it is sometimes viewed as a form of demonic possession, especially if it has a dramatic manifestation such as a full-blown epileptic seizure, or schizophrenia, but generally, déjà vu in religious context is not seen as something "bad", but a distinguishing feature, a transcending experience connecting a person to the higher realms. In spite of many definitions and theories, déjà vu still remains a mystery, startling the minds of countless scientists and laymen, since new studies and equipment that we still do not possess are needed in order to reveal its true origin, as well as its meaning (Horvat Velić 2017).

\section{NEUROBIOLOGY OF DÉJÀ VU}

It seems that temporal region is the origin of déjà vu phenomena in healthy individuals and in individuals with neurological and psychiatric conditions, but the exact mechanism as well as the precise anatomical basis of this phenomenon still remains unknown.

Three structures are clearly associated with déjà vu experiences as a part of a neurological conditions (hippocampus, parahippocampal gyrus, and temporal neocortex). The most serious of those conditions is surely temporal lobe epilepsy where prolonged duration and higher occurrence frequency have been indicators of a said pathophysiology, when compared to otherwise non-affected brains (Sno et al. 1992).

Pathophysiological hypotheses of déjà vu in epilepsy, as Chauvel reviewed, involved either the limbic regions of the temporal lobe, the temporal neocortex or both of them. New hypothesis of corticolimbic network has emerged, suggesting that déjà vu could result from an abnormal synchronisation between rhinal cortices and hippocampus (see Chauvel 2014). Namely, Bartolomei et al. studied the role of perirhinal and enthorinal cortices in déjà vu in patients with epilepsy and analysed the symptoms evoked by direct electrical stimulations of abovementioned regions in comparison to stimulation of amygdala and hippocampus. Their results demonstrated that stimulation of perirhinal and enthorinal cortices had more often produced déjà vu responses, with enthorinal cortex being the most sensitive site. They emphasized that findings from previous studies, reporting déjà vu after stimulation of amygdala or hippocampus, might be explained by provocation of déjà vu by spread of the discharge from those regions to the adjacent rhinal cortex (Bartolomei et al. 2004). Further analysis by Bartolomei et al., where familiarity experience occurred only when the EEG signal correlation increased between rhinal cortices, amygdala and hippocampus, seems to confirm that déjà vu phenomenon could be related to more than one system with abnormal synchronisation between them (Chauvel 2014, Bartolomei et al. 2012).

Regarding structural anatomy of déjà vu, when Brázdil et al. investigated brain morphology in healthy subjects with and without déjà vu experiences, they found differences in grey matter volume between these two groups in some regions of the brain, with significantly less grey matter in subjects reporting déjà vu accompanied with an inverse correlation between grey matter volume in those regions and the frequency of déjà vu experiences. Reported regions included bilateral mesiotemporal regions (with maximal effect within hippocampi and parahippocampal gyri), insular cortices, superior temporal sulci, basal ganglia and thalami (Brázdil et al. 2012).

Studying the effects of psychotropic drugs and medication which are described to be associated with déjà vu in literature, could lead us closer to uncovering the 
origins of déjà vu phenomena. For instance, Singh reported Salvia divinorum as a possible cause of déjà vu in a young male with history of smoking salvia (Singh 2007), while Taiminen and Jääskeläinen reported recurrent déjà vu experiences in 39-year old healthy male within $24 \mathrm{~h}$ of initiating concomitant amantadine-phenylpropanolamine treatment against influenza (Taiminen \& Jääskeläinen 2001).

\section{DÉJÀ VU IN PSYCHIATRY}

Déjà vu is not a meaningful symptom in psychiatry, although the response to it by patients with schizophrenia may be more pronounced then in healthy population, and patients with anxiety and people with derealisation/depersonalization tend to experience it more often than healthy groups. Because of a neurobiological model of depersonalization involving the same limbictemporal networks as in epilepsy syndromes which relate to déjà vu aura, it seems that exploration of the déjà state in depersonalization is important for future research in psychiatry too (O'Connor \& Moulin 2010).

Patients suffering from schizophrenia have an altered experience of time, and construct their reality in larger time fragments, which should be taken into account when treating them and assessing their ability to perceive the reality around them. Among the altered experiences of time are exactly déjà vu and déjà vecu, with notably different reactions to the experiences (Stanghellini et al. 2016, Sengoku et al. 1997). Déjà vu is usually associated with feelings of pleasantness, while déjà vecu is usually associated with feelings of fear and anxiety (Sengoku et al. 1997). Although altered experiences of time were predominantly studied in patients suffering from schizophrenia, there is no statistical significance in altered time experiences in patients with other psychiatric conditions (Stanghellini et al. 2016).

Another interesting finding, which warrants further research, is appearance of psychotic symptoms in a patient who reported to an emergency room after smoking Salvia divinorum, a hallucinogen plant from the Lamiaceae order. After subsiding of initial paranoid and panic symptoms, déjà vu experiences remained and he reported them a significant time after the expected termination of salvia effects. After ruling out other possible causes of déjà vu experiences, it was concluded that salvia was the most likely cause of déjà vu in the patient (Singh 2007).

\section{Déjà vu and false memories}

The connection between false memories formation and déjà vu in healthy individuals has been found by using hypnosis (O'Connor et al. 2008), fMRI (Chadwick et al. 2016) and EEG (Sederberg et al. 2007). In false memory creation, certain areas of the brain (hippocampus, temporal and prefrontal cortex) play a significant role.
Hypnosis has the potential to provide a reliable laboratory analogue of déjà vu. Group of authors created a laboratory analogue of déjà vu consistent with our current understanding of déjà vu, involving false familiarity for an unfamiliar stimulus. Puzzle game was played in one group but suggested to forget about it, and in other group the game was not played, but it was suggested to feel familiar about it. More people who, in fact, did not play the game reported sense of (false) familiarity because of hypnotic suggestion (O'Connor et al. 2008). This study connects false memories with aetiology of déjà vu in otherwise healthy people.

Using fMRI, Chadwick et al. discovered that false memories emerge from a similarity-based neural code in the temporal pole, a region that has been called the "semantic hub" of the brain. They also found that each individual has a partially unique semantic code within the temporal pole, and this unique code can predict idiosyncratic patterns of memory errors (Chadwick et al. 2016).

Group of authors (Sederberg et al. 2007) used EEG to distinguish true from false memories and found increased gamma oscillations immediately preceding a response. Recall of a true memory is preceded by an increase in gamma oscillations in the hippocampus (bilaterally) and in the temporal and prefrontal cortices (primarily in the left hemisphere) (Sederberg et al. 2007).

\section{DÉJÀ VU IN NEUROLOGY}

\section{Déjà vu and temporal lobe epilepsy}

Temporal lobe epilepsy (TLE) is epilepsy in which seizures start in the temporal lobe of the brain. There are currently two different types of TLE, with each based on the anatomical division of their origin points. First type, mesial temporal lobe epilepsy, originates in the medial parts of the temporal lobe (structures included: hippocampus, parahippocampal gyrus and amygdala). Lateral temporal lobe epilepsy (LTLE), as the name suggests, originates in the neocortex of the temporal lobe. Moreover, mesial temporal lobe epilepsy is the most common type of epilepsy found in humans (Engel 2001), and can be further divided into three different subtypes, in accordance with different symptoms: simple partial seizures (SPS), complex partial seizures, and secondarily generalized tonic-clonic seizures. Precisely simple partial seizures relate to the most of déjà experiences (Illman et al. 2012).

On the other hand, Adachi et al. showed in 2010 that things might not be so simple. They devised a special scale called Inventory of Déjà vu Experiences Assessment in order to measure déjà vu experiences in participants. As a part of the study they examined 402 healthy subjects and 312 patients who suffered from temporal lobe epilepsy (Adachi et al. 2010). The results were rather conflicting with previous knowledge - it showed that $76.1 \%$ of healthy individuals experienced déjà vu phenomenon, as well as $63.1 \%$ of the patients 
with epilepsy experiencing the same (Adachi et al. 2010). It was suggested, based on this research, that déjà vu experiences might not be sign of a pathological state of the brain, but rather a normal occurrence in everyday life. Despite all this, the consensus is still that déjà vu is more persistent in patients who exhibit the experiences as the part of simple partial seizures. It is important to consider that familiarity based memory error is the likely cause for the formation of déjà vu experience in healthy individuals, which leads to conclusion that déjà vu occurring in simple partial seizures does not form the same as in healthy individuals (Illman et al. 2012). As hippocampus and parahippocampal gyrus are structures involved in the formation of new memories, and they also play the vital role in the process of recognition of scenes and places, it is believed that the formation of déjà vu experience is directly connected to those brain parts (Illman et al. 2012).

However, some argue that déjà vu experience is a product of "a false activation of connections between mesiotemporal memory structures and neocortical areas directly involved in the perception of the environment" (Spatt 2002). It is concluded that false activation produces the feeling of familiarity of the new place or new situation, and it is thought this event occurs due to the activation of parahippocampal gyrus, with hippocampus not playing any part in the process. That hypothesis is somewhat confirmed in the experimental studies in which epileptiform activity was found in parahippocampal gyrus, as well as a study in which neocortical temporal regions connected to parahippocampal gyrus were stimulated. Two studies were conducted, as described by Spatt J 2002, and it was suggested that déjà vu experience in healthy individuals may be "a by-product of a mechanism that is responsible for memory consolidation" which is at its peak during sleep (Spatt 2002).

On the other hand, it seems that amygdala pathology alone can be involved in pathological déjà vu (together with olfactory auras), as described in the case of a teenager who presented with seizures and déjà vu as well as olfactory auras that resolved following amygdalectomy (Lee et al. 2009).

The exact mechanisms and reasons for déjà vu experience are still unclear, but with the help of EEG intracranial monitoring, three theories for dysfunctions have been proposed: dysfunction in mesial temporal lobe of the non-dominant hemisphere, the superior lateral temporal cortex and neuronal network that engages both mesial and lateral parts of the temporal lobe (Panayiotopoulos 2005). However, Adachi et al. in their aforementioned study suggested that other areas of the brain are also responsible in the formation of the déjà vu event (Adachi et al. 2010). In addition, study by Vlasov et al conducted in 2013 clearly distinguishes between epileptic and non-epileptic déjà vu with clear conclusion that déjà vu can be a part of a secondarily generalized seizure, or regarded as an equivalent to a simple partial seizure. It is frequently followed by the feelings of fear, and it occurs more often than in the healthy subjects (Vlasov et al. 2013).

An interesting case of temporal lobe epilepsy accompanied by déjà vu experiences was reported by Ide et al. of a patient with a history of psychomotor seizures (Ide et al. 2000). During the treatment of the seizures with sodium valproate the patient started experiencing déjà vu episodes. The patient underwent SPECT as a mean of localizing the epileptiform lesions in her brain. After some time, clonazepam was added to her therapy, and after increasing the dosage of clonazepam her déjà vu experiences subsided. The patient kept having psychomotor seizures, but without further déjà vu episodes. What is interesting about this case is a subsequent SPECT finding of improved hypoperfusion in her brain lesions associated with psychomotor seizures and the authors concluded that clonazepam was the likely cause despite there being no known effects of clonazepam on brain perfusion (Ide et al. 2000).

\section{Déjà vu and other neurological conditions}

The study that took place from 2008 to 2013 had the aim of describing different features of migraine aura in teenage patients in detail (Petrusic et al. 2014). Noted symptoms were visual aura, scintillating scotoma, blurry vision and symptoms of higher cortical dysfunction (HCD), with slowed speech. However, déjà vu experiences were found in $22.5 \%$ of the patients that participated, and déjà vu itself is considered as one of the HCD symptoms (Petrusic et al. 2014). Cortical spreading depression (CSD), defined as a wave of electrophysiological activity that originates strictly in the occipital region has also been shown to have déjà vu symptoms. CSD is for now mostly connected and found in the sufferers of chronic migraines (Petrusic \& Zidverc-Trajkovic 2014). In addition, déjà vu experiences were found in patients that suffer from vestibular disease, in correlation with depersonalisation and derealisation symptoms (Sang et al. 2006).

\section{CONCLUSION}

Déjà vu is obviously common in the population as a normal psychological event, but it can also be a valuable indicator of many pathophysiological states of the brain (temporal lobe epilepsy and migraine being the most prominent). It is still very much unknown phenomenon, with many studies conducted in the past and many more in plans for future research. This mystery of déjà vu could be revealed only with multidisciplinary approach through cooperation between neurobiologists, neurologists, brain scientists, psychiatrists and experimental psychologists, for example in a form of international collaborations such as the BRAIN Initiative and Blue Brain Project, which will surely drive us closer up to that goal. 


\section{Acknowledgements: None.}

\section{Conflict of interest: None to declare.}

\section{Contribution of individual authors:}

Marija Bošnjak Pašić made substantial contributions to conception and design of this review, interpretation of data and also participated in drafting the article, revising it and giving the final approval of the version to be submitted.

Emina Horvat Velić, Luka Fotak, Hanna Pašić and Sarah Bjedov were involved in literature searches, interpretation of data and drafting the article.

Azijada Srkalović Imširagić and Davorka Milat participated in interpretation of data, drafting and revising of the article.

Helena Šarac and Željka Petelin Gadže were involved in interpretation of data, drafting the article, critically revising of the manuscript for important intellectual content and giving the final approval of the version to be submitted.

\section{References}

1. Adachi N, Akanuma N, Ito M, Adachi T, Takekawa Y, Adachi $Y$ et al.: Two forms of déjà vu experiences in patients with epilepsy. Epilepsy Behav 2010; 18:218-22

2. Bartolomei F, Barbeau E, Gavaret $M$, Guye $M$, McGonigal A, Régis J et al.: Cortical stimulation study of the role of rhinal cortex in déjà vu and reminiscence of memories. Neurology 2004; 63:858-64

3. Bartolomei F, Barbeau EJ, Nguyen T, McGonigal A, Régis $J$, Chauvel $P$ et al.: Rhinal-hippocampal interactions during déjà vu. Clin Neurophysiol 2012; 123:489-95

4. Brázdil M, Mareček R, Urbánek T, Kašpárek T, Mikl M, Rektor I et al.: Unveiling the mystery of déjà vu: the structural anatomy of déjà vu. Cortex 2012; 48:1240-3

5. Chauvel P: Pathophysiology of Déjà Vu and Reminiscences in Epilepsy. P Belg Roy Acad Med 2014; 3:104-113

6. Chadwick MJ, Anjum RS, Kumaran D, Schacter DL, Spiers HJ \& Hassabis D: Semantic representations in the temporal pole predict false memories. Proc Natl Acad Sci USA 2016; 113:10180-5

7. Engel J: Mesial temporal lobe epilepsy: what have we learned? Neuroscientist 2001; 7:340-52

8. Horvat Velić E: The mystery of déjà vu. Gyrus 2017; 4:97-100

9. Ide M, Mizukami K, Suzuki T \& Shiraishi H: A case of temporal lobe epilepsy with improvement of clinical symptoms and single photon emission computed tomography findings after treatment with clonazepam. Psychiatry and Clinical Neurosciences 2000; 54:595-597
10. Illman NA, Butler CR, Souchay C\& Moulin CJA: Déjà Experiences in Temporal Lobe Epilepsy. Epilepsy Res Treat 2012; 2012:539567; doi:10.1155/2012/539567

11. Lee DJ, Owen CM, Khanifar E, Kim RC\& Binder DK: Isolated amygdala neurocysticercosis in a patient presenting with déjà vu and olfactory auras. $J$ Neurosurg Pediatr 2009; 3:538-541

12. Neppe VM: An Overview Perspective on what Déjà Vu is (Part 1). J Psychol Clin Psychiatry 2015; 2:00111

13. O'Connor AR, Barnier AJ \& Cox RE: Déjà vu in the laboratory: a behavioral and experiential comparison of posthypnotic amnesia and posthypnotic familiarity. Int $J$ Clin Exp Hypn 2008; 56:425-50

14. O'Connor AR \& Moulin CJ: Recognition without identification, erroneous familiarity, and déjà vu. Curr Psychiatry Rep 2010; 12:165-73

15. Panayiotopoulos CP: The Epilepsies: Seizures, Syndromes and Management. Bladon Medical Publishing, Oxfordshire (UK), 2005

16. Petrusic I, Pavlovski V, Vucinic D \& Jancic J: Features of migraine aura in teenagers. J Headache Pain 2014; 15:87

17. Petrusic I \& Zidverc-Trajkovic J: Cortical spreading depression: origins and paths as inferred from the sequence of events during migraine aura. Funct Neurol 2014; 29:207-212

18. Sang FYP, Jáuregui-Renaud K, Green DA, Bronstein AM \& Gresty MA: Depersonalisation/derealisation symptoms in vestibular disease. J Neurol Neurosurg Psychiatry 2006; 77:760-6

19. Sederberg PB, Schulze-Bonhage A, Madsen JR, Bromfield EB, Litt B, Brandt $A$ et al.: Gamma Oscillations Distinguish True From False Memories. Psychol Sci 2007; 18:927-932

20. Sengoku A, Toichi $M$ \& Murai T: Dreamy states and psychoses in temporal lobe epilepsy: Mediating role of affect. Psychiatry Clin Neurosci 1997; 51:23-26

21. Singh $S:$ Adolescent salvia substance abuse. Addiction 2007; 102:823-824

22. Sno HN, Linszen DH \& de Jonghe F: Art imitates life: Déjà vu experiences in prose and poetry. Br J Psychiatry 1992; 160:511-8

23. Spatt J: Déjà vu: possible parahippocampal mechanisms. J Neuropsychiatry Clin Neurosci 2002; 14:6-10

24. Stanghellini $G$, Ballerini $M$, Presenza S, Mancini M, Raballo A, Blasi $S$ et al.: Psychopathology of Lived Time: Abnormal Time Experience in Persons With Schizophrenia. Schizophr Bull 2016; 42:45-55

25. Taiminen $T \&$ Jääskeläinen SK: Intense and recurrent déjà vu experiences related to amantadine and phenylpropanolamine in a healthy male. J Clin Neurosci 2001; 8:460-2

26. Vlasov PN, Chervyakov AV \& Gnezditskii VV: Déjà vu phenomenon-related EEG pattern. Case report. Epilepsy Behav Case Rep 2013; 1:136-141

Correspondence:

Sarah Bjedov, $M D$

University Hospital Centre Zagreb, Department of Psychological Medicine

Kišpatićeva 12, 10000 Zagreb, Croatia

E-mail:sarahbjedov@gmail.com 